\title{
Vaccination of mice for research purpose: alum is as effective as and safer than complete Freund adjuvant
}

\author{
N. Bassi ${ }^{1}$, R. Luisetto ${ }^{2}$ A. Ghirardello', M. Gatto', B. Bottazzi ${ }^{3}$, \\ Y. Shoenfeld ${ }^{4}$, L. Punzi ${ }^{1}$, A. Doria ${ }^{1}$ \\ ${ }^{1}$ Department of Medicine, Division of Rheumatology, University of Padua, Italy; \\ 2Department of Medicine, Division of Experimental Surgery, University of Padua, Italy; \\ ${ }^{3}$ Department of Translational Medicine, University of Milan, Italy; \\ ${ }^{4}$ Department of Medicine B, Center for Autoimmune Diseases, Sheba Medical Center, \\ University of Tel-Aviv, Tel-Hashomer, Israel
}

Work awarded at the XLVIII Congress of the Italian Society of Rheumatology (SIR), Rimini 2011

\section{SUMMARY}

Systemic lupus erythematosus (SLE) is an autoimmune disease involving many organ systems. Glomerulonephritis (GLN) is one of the major causes of morbidity and mortality in SLE. It has recently been demonstrated that adjuvants of vaccines could cause the so called ASIA syndrome. The study aimed to assess the effects of Complete Freund's Adjuvant (CFA) vs alum injections in NZB/NZWF1 mice. Mice ( $\mathrm{n}=10$ each group) were injected with a total volume of $200 \mu \mathrm{L}$ of: CFA in PBS (group 1), alum in PBS (group 2), PBS (group 3) as controls, PTX3/CFA (group 4), PTX3/alum (group 5), 3 times, 3 weeks apart /given in each injection, three weeks apart from ten weeks of age. Urine samples were collected weekly to evaluate proteinuria. Blood samples were collected before every injection, at 21 weeks of age, and at death to evaluate levels of anti-PTX3 and anti-dsDNA. Proteinuria free survival and survival rates were analyzed by the Kaplan-Meier method using Mantel-Cox's test for comparisons.

CFA-treated mice developed both anti-dsDNA antibodies and proteinuria earlier and at higher levels than alumtreated and PBS-injected mice, starting from 13 weeks of age. Proteinuria free survival rates (proteinuria $\geq 300$ $\mathrm{mg} / \mathrm{dL}$ ) and survival rates were lower in CFA-treated mice than those treated with alum or injected with PBS ( $\mathrm{P}<0.001$ for all). No difference was observed between the alum-treated group and PBS-injected mice. Notably, groups 4 and 5, immunized with PTX3, developed anti-PTX3 antibodies and no significant difference was observed.

Alum seems to be as effective as and safer than CFA as adjuvant, since it did not affect disease progression in immunized NZB/NZWF1 mice.

Key words: Systemic lupus erythematosus (SLE), glomerulonephritis (GLN), NZB/NZWF1 mice, ASIA syndrome, adjuvants.

Reumatismo, 2012; 64 (6): 380-387

\section{INTRODUCTION}

ystemic lupus erythematosus (SLE) $\checkmark$ is a systemic, multifactorial, autoimmune disease involving many organ systems. SLE pathogenesis involves genetic predisposition, defective apoptosis and abnormalities in Toll-like receptor (TLR) signaling, B and T-cell tolerance, complement activation, cytokine regulation, and endothelial cells both in humans and in mice (13 ). All these factors cause the production of autoantibodies, mainly antinucleosome and anti-double stranded DNA (dsDNA), which can be found deposited in tissues as immune complexes (ICs) $(4,5)$. One of the major causes of morbidity and mortality in SLE is glomerulonephritis (GLN) that affects $40-60 \%$ of patients (5) and leads to chronic renal failure in $25 \%$ of cases (6). Many studies show that vaccinations may trigger or worsen autoimmune and rheumatic diseases because of the release of proinflammatory cytokines after the injection (7-9), resulting in the stimulation of innate and adaptive immune response with increased activation, proliferation and migration of dendritic cells, macrophages, $\mathrm{T}$ and $B$ cells to the injection site. Notably, not only the antigens of the vaccines, but also 
adjuvants and excipients may enhance antigenic stimulation $(7,10,11)$. In humans, vaccines have been reported to potentially induce autoantibodies, inflammatory reactions, and autoimmune diseases, such as arthritis, neuronal damage, fatigue, encephalitis, myocarditis, and vasculitis (12, 13). These adverse events can occur weeks or even months or years after vaccination (13). Mineral oils may induce sclerosing lipogranulomas in humans (14). Alum, aluminium hydroxide and squalene are all reported as cofactors in causing chronic fatigue syndrome (CFA), polymyalgia, macrophagic myofasciitis syndrome (MMS), and Gulf War syndrome (GWS) $(15,16)$. Adjuvants may also induce the production of antibodies against themselves, as reported in US military personnel with GWS, in whom high levels of anti-squalene circulating antibodies were found (16-18).

Many in vivo studies show that adjuvants can induce autoimmunity also in animal models (19-21). Subcutaneous injection of mineral oil induced sclerosing lipogranulomas not only in humans but also in $\mathrm{BALB} / \mathrm{c}$ mice (14). Pristane $(2,6,10,14$ - tetramethylpentadecane) and mineral oil mixtures induced plasmacytomas or lupusrelated autoantibodies to nRNP/Sm and to $\mathrm{Su}$ in $\mathrm{BALB} / \mathrm{c}$ mice $(21,22)$. Mice and rats treated with squalene $(2,6,10,15,19,23$ - hexamethyl - 2, 6, 10, 14, 18, 22-tetracosahexaene) developed arthritis (23).

It has recently been proposed to group syndromes developed as a consequence of vaccinations, such as MMF or GWS, under the common syndrome called Autoimmune (Autoinflammatory) Syndrome Induced by Adjuvants (ASIA) (24). We have recently demonstrated that the injection of complete Freund's adjuvant (CFA), one of the most commonly used adjuvants because of its composition including heat-killed and dried Mycobacterium tuberculosis, paraffin oil, and mannide mono-oleate, causes the onset of the ASIA syndrome in NZB/ NZWF1 mice (25). NZB/NZWF1 mice are a murine model of human SLE and develop a lupus-like GLN within 5-7 months of age (3). As in humans, anti-dsDNA antibodies are found at high levels in the circula- tion and deposited as ICs in glomeruli (3). The aim of our study was to evaluate whether CFA might interfere during vaccinations, nullifying the therapeutic beneficial effect of vaccines because of the development of an exaggerated immune response. The study also aimed to evaluate whether alum could be safer than CFA and as efficacious as CFA for vaccinations.

\section{MATERIALS AND METHODS}

Eight-week old NZB/NZWF1 mice (Harlan Laboratories, Indianapolis, IN, USA) were divided into 5 groups of 10 mice each and injected with a total volume of $200 \mu \mathrm{L}$ of: $100 \mu \mathrm{L}$ CFA (Sigma, St Louis, MO, USA) in $100 \mu \mathrm{L}$ PBS (group 1); 100 $\mu \mathrm{L}$ alum (Sigma) in $100 \mu \mathrm{L}$ PBS (group 2); PBS (group 3), as controls; $100 \mu \mathrm{g} / \mathrm{mL}$ of long pentraxin 3 (PTX3) in CFA (group 4); $100 \mu \mathrm{g} / \mathrm{mL}$ PTX3 in alum (group 5); given in each injection, three weeks apart, starting from 10 weeks of age to 16 . All mice were bred until natural death occurred. The study was approved by the Institutional Animal Care and Use Committee.

Disease progression was monitored by weekly collection of urine samples in order to evaluate proteinuria levels using multi reactive strips (Bayer, Leverkusen, Germany) and expressed as mg/dL. Blood samples were collected from the caudal vein before every injection (at 10,13 and 16 weeks of age), at week 21, and at death. Circulating levels of anti-dsDNA were evaluated in all groups, whereas those of anti-PTX3 were evaluated only in groups 4 and 5; standardized home-made ELISA tests were used in both groups. For antidsDNA ELISA tests, the coating was performed in 3 phases: addition of poly-Llysine to block the DNA and incubation at $37^{\circ} \mathrm{C}$, addition of calf thymus DNA and incubation at room temperature (RT), and addition of poly-L-glutamate to neutralize the negative free charges of DNA and incubation at RT. After washing circles, 3\% BSA/TBS was added as blocker and incubated overnight at $4^{\circ} \mathrm{C}$. Duplicated serum samples were added at a dilution of 1:100 
in $1 \% \mathrm{BSA} / \mathrm{TBS}$ and incubated at RT. After washings, anti-mouse IgG (diluted $1: 10000$ in $1 \%$ BSA/TBS) was added and incubated at $37^{\circ} \mathrm{C}$. After washing circles, $p$-nitrophenil-phosphate was added and the plates were read at $405 \mathrm{~nm}$. Anti-PTX3 antibody levels were evaluated as previously described by Bassi et al. (26). Briefly, recombinant PTX3 was obtained by purification from transfected $\mathrm{CHO}$ cells according to Bottazzi et al. (27). Plates were coated with recombinant PTX3 diluted in PBS and incubated overnight at $4^{\circ} \mathrm{C}$. Blocking was performed with $3 \% \mathrm{BSA} / \mathrm{PBS}$, incubated at RT. Duplicated serum samples were added at a dilution of 1:100 in 1\% BSA/PBS and incubated at RT. After washings, anti-mouse $\mathrm{IgG}$, diluted 1:10000 in $1 \% \mathrm{BSA} / \mathrm{PBS}$, was added and incubated at $37^{\circ} \mathrm{C}$. After washing circles, $p$-nitrophenylphosphate was added and the plates were read at $405 \mathrm{~nm}$. All products for ELISA tests were purchased from Sigma, St Louis, MO, USA. The values were expressed as mean optical density (OD) of the double of every serum sample.

\section{Statistical analyses}

Statistical analyses were performed using PASW 18 software. Differences between groups were evaluated by Mann-Whitney test; survival rates and the onset of critical proteinuria levels (at least $300 \mathrm{mg} / \mathrm{dL}$ ) were evaluated by Kaplan-Meyer method using Mantel-Cox's test for comparison.

\section{RESULTS}

\section{Comparison between CFA and alum ef- fects}

Mice of groups 1, 2 and 3 developed antidsDNA autoantibodies. However, in group 1, anti-dsDNA antibodies were detectable earlier than in mice of the other 2 groups. In fact, group 1 developed anti-dsDNA antibodies starting from 13 weeks of age after only one injection, whereas the PBS-injected group developed anti-dsDNA antibodies at 16 and group 2 mice at 21 weeks of age. The circulating levels of anti-dsDNA, expressed as mean $\mathrm{OD} \pm$ standard deviation
(SD), were higher in CFA-treated mice compared with alum-treated mice: 13 weeks $0.397 \pm 0.19$ vs $\quad 0.110 \pm 0.03, \quad \mathrm{P}<0.0001$; 16 weeks $0.535 \pm 0.17$ vs $0.165 \pm 0.06$, $\mathrm{P}<0.0001 ; \quad 21$ weeks $0.728 \pm 0.19 \quad v s$ $0.395 \pm 0.05, \mathrm{P}=0.007$ (Fig. 1A). Notably, CFA-treated mice had higher levels than PBS-injected mice: 13 weeks $0.397 \pm 0.19$ vs $\quad 0.151 \pm 0.04, \quad \mathrm{P}<0.0001 ; 16$ weeks $0.535 \pm 0.17$ vs $0.237 \pm 0.09, \mathrm{P}<0.0001 ; 21$ weeks $0.728 \pm 0.19$ vs $0.508 \pm 0.10, \mathrm{P}=0.005$. No significant difference was observed between group 2 and group 3: 13 weeks $0.110 \pm 0.03$ vs $0.151 \pm 0.04, \quad \mathrm{P}=$ n.s.; 16 weeks $0.165 \pm 0.06$ vs $0.237 \pm 0.09, \mathrm{P}=\mathrm{ns}$; 21 weeks $0.395 \pm 0.05$ vs $0.508 \pm 0.10, \mathrm{P}=\mathrm{ns}$ (Fig. 1B).

Similarly, group 1 mice developed proteinuria earlier and in higher amounts than the other 2 groups. In fact, 6 out of 10 CFAtreated mice developed traces of proteinuria $(15 \mathrm{mg} / \mathrm{dL})$ at 13 weeks of age and the last 4 at 16 weeks of age. In groups 2 and 3 , traces of proteinuria occurred in the first mouse at 19 weeks of age. Proteinuria levels, expressed as mean $(\mathrm{mg} / \mathrm{dL}) \pm \mathrm{SD}$, were higher in CFA-treated mice compared with alum-treated mice: 13 weeks $15 \pm 14.14$ vs $0, \mathrm{P}=0.001 ; 16$ weeks $73.50 \pm 88.07$ vs $0, \mathrm{P}=0.010 ; 19$ weeks $610.00 \pm 417.53$ vs $10.50 \pm 10.12, \quad \mathrm{P}<0.0001 ; 21$ weeks $720 \pm 383.41$ vs $18.00 \pm 13.78, \mathrm{P}=0.003$ (Fig. 1C). Proteinuria levels were significantly higher in CFA-treated mice compared to PBS-injected mice: 13 weeks $15 \pm 14.14$ vs $0, \mathrm{P}=0.001 ; 16$ weeks $73.50 \pm 88.07$ vs $0, \mathrm{P}=0.010 ; 19$ weeks $610.00 \pm 417.53 v s$ $18 \pm 13.74, \mathrm{P}<0.0001 ; 21$ weeks $720 \pm 383.41$ vs $27 \pm 6.33, \mathrm{P}<0.0001$; whereas the levels in groups 2 and 3 were comparable: 13 weeks 0 vs $0, \mathrm{P}=\mathrm{ns} ; 16$ weeks 0 vs $0, \mathrm{P}=\mathrm{ns}$; 19 weeks $10.50 \pm 10.12$ vs $18 \pm 13.74, \mathrm{P}=\mathrm{ns}$; 21 weeks $18.00 \pm 13.78$ vs $27 \pm 6.33, \mathrm{P}=\mathrm{ns}$ (Figure 1D). Notably, all mice in group 1, 5 in group 2, and 6 in group 3 died after developing the maximum detectable levels of proteinuria $(\geq 300 \mathrm{mg} / \mathrm{dL})$.

Proteinuria free survival rate (proteinuria $<300 \mathrm{mg} / \mathrm{dL}$ ) was significantly lower in group 1 than in groups 2 and $3(\mathrm{P}<0.0001$ for all) (Fig. 2A). At 20 weeks of age, $90 \%$ of the mice in group 1 had proteinuria lev- 


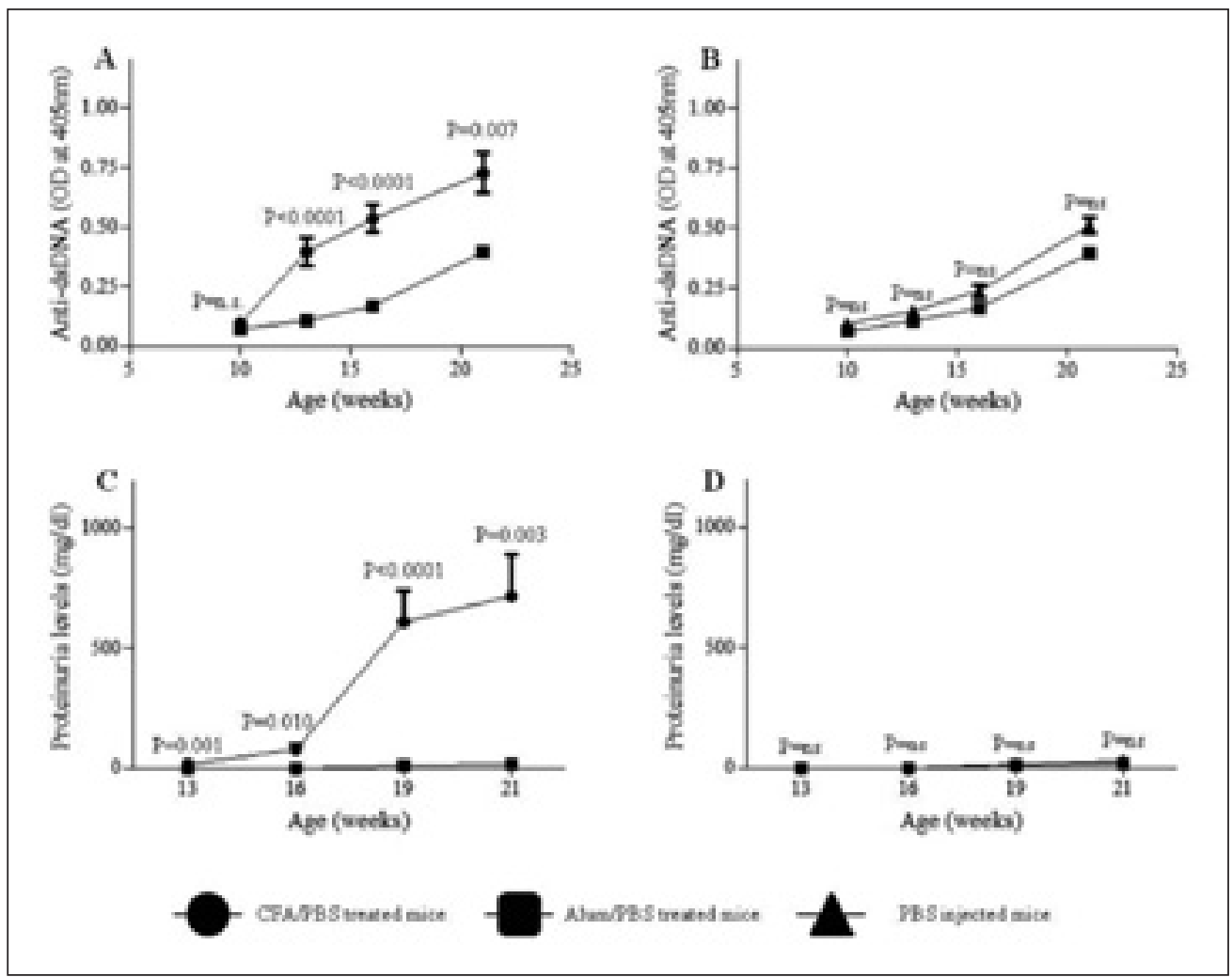

Figure 1 - Comparison of (A) mean anti-dsDNA autoantibodies levels (OD at $405 \mathrm{~nm}$ ) between mice treated with CFA/PBS and those injected with alum/PBS and (B) between alum/PBS-injected mice and PBS-injected mice. (C) Mean proteinuria levels $(\mathrm{mg} / \mathrm{dL})$ between mice treated with CFA/PBS and those injected with alum/PBS and (D) between alum/PBS-injected group and PBS-injected group. dsDNA, double stranded DNA; OD, optical density; CFA, complete Freund's adjuvant; PBS, phosphate buffer salt; ns, not significant.

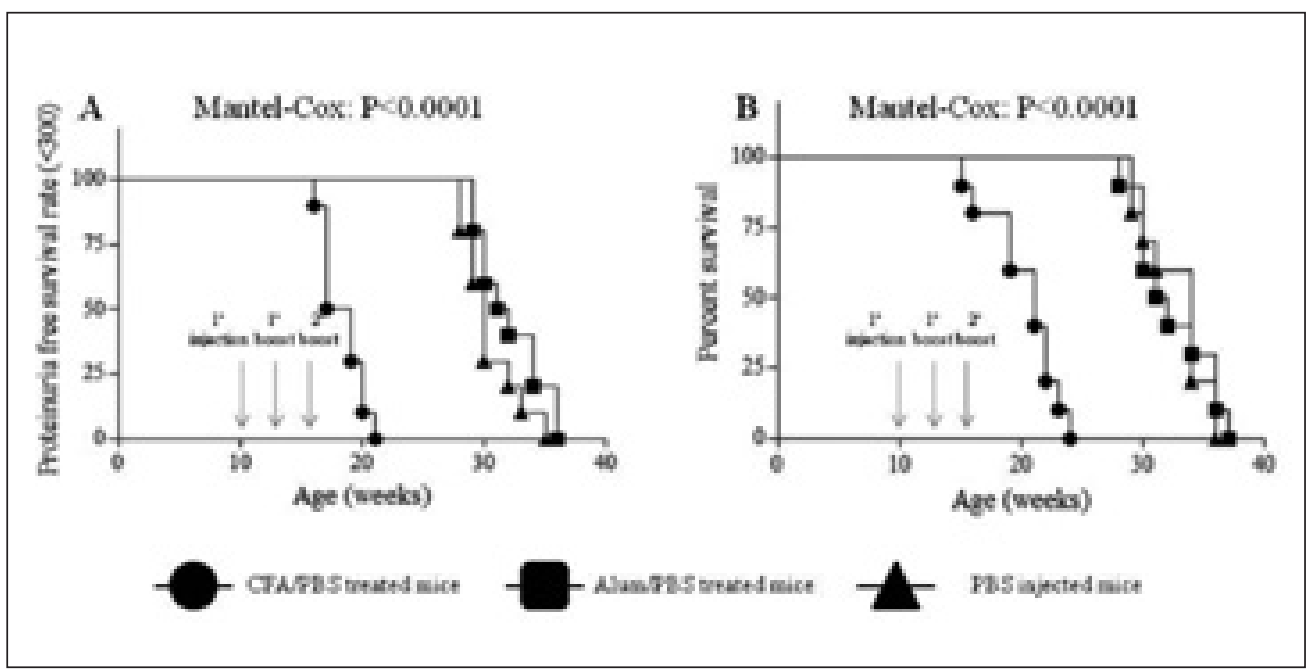

Figure 2 - Comparison of $(A)$ proteinuria free survival rate $(<300 \mathrm{mg} / \mathrm{dL})$ between mice treated with CFA/PBS and alum/PBS and PBS. (B) Survival rate between mice treated with CFA/PBS and alum/PBS and PBS. CFA, complete Freund's adjuvant; PBS, phosphate buffer salt. 


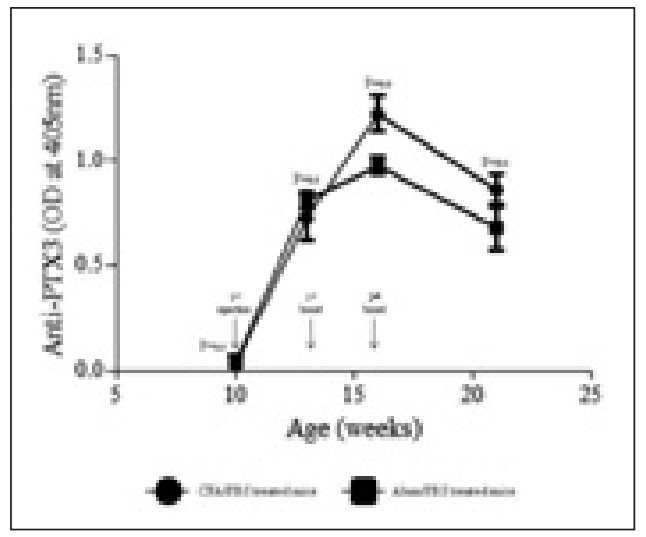

Figure 3 - Comparison between mice treated with PTX3/CFA and those injected with PTX3/ alum: mean anti-PTX3 autoantibody levels (OD at $405 \mathrm{~nm}$ ). CFA, complete Freund's adjuvant; PTX3, long pentraxin 3; OD, optical density; ns, not significant.

els of $300 \mathrm{mg} / \mathrm{dL}$ or over, whereas the first of PBS and alum-injected mice developed these levels of proteinuria at 28 and 29 weeks of age, respectively $(\mathrm{P}<0.0001$ for both). Proteinuria of $300 \mathrm{mg} / \mathrm{dL}$ or over occurred at 21 weeks of age in the last mouse of the CFA-injected group and at 35 and 36 weeks of age in the last mouse of the PBS and alum-injected groups, respectively. Mean proteinuria free survival rate (mean weeks \pm SD) was significantly lower in CFA-treated mice than in those treated with alum $(18.3 \pm 1.7$ vs $32.1 \pm 2.7, \mathrm{P}<0.0001)$ or in those injected with PBS $(18.3 \pm 1.7$ vs $30.4 \pm 2.2, \mathrm{P}<0.0001)$. No significant difference was observed between groups 2 and 3 (32.1 \pm 2.7 vs $30.4 \pm 2.2, \mathrm{P}=\mathrm{ns})$.

Survival rate was significantly lower in group 1 than in groups 2 and 3 (Fig. 2B). The first death in group 1 was recorded in a 15 -week old mouse, whereas the first death in group 2 occurred in a 28 -week old mouse and in group 3 in a 29-week old mouse. The last death in the CFA-treated group occurred in a 24-week old mouse. By contrast, the last death in the PBSinjected group occurred in 2 36-week old mice, and in the alum-treated group the last death occurred in a 37-week old mouse. At 21 weeks of age, the survival rate was $40 \%$ in group $1 \%$ vs $100 \%$ in groups 2 and 3 . When the last mouse in the CFA-treated group died, all mice of PBS or alum-injected group were still alive $(\mathrm{P}<0.0001)$ (Fig. 2B). Mean survival rate (mean weeks \pm SD) was significantly lower in CFA-treated mice than in those treated with alum (group $1 v s$ group 2: 20.2 $\pm 2.9 v s$ 32.7 \pm 3.1 , $\mathrm{P}<0.001)$ or in those injected with PBS (group 1 vs group 3: 20.2 \pm 2.9 vs 32.4 2.7 , $\mathrm{P}<0.0001)$. No significant difference was observed between groups 2 and 3 (group 2 vs group 3: $32.7 \pm 3.1$ vs $32.4 \pm 2.7, \mathrm{P}=\mathrm{ns}$ ).

\section{Comparison between vaccination with CFA and with alum}

Notably, no difference was observed in the anti-PTX3 antibody levels between PTX3/ CFA and PTX3/alum treated mice (Fig. 3). In fact, both groups developed anti-PTX3 antibodies starting from 13 weeks of age, after only one injection, and the maximum amount was observed at 22 weeks of age and then decreased in both groups, always with comparable mean values (Fig. 3).

\section{DISCUSSION}

In our experiment, all NZB/NZWF1 mice receiving CFA demonstrated a worsening of autoimmune response along with a worsening of GLN and a shortening of survival time in comparison with those receiving alum and the control group, whereas no difference was observed between alumtreated and PBS-injected mice. In fact, all mice in the CFA-treated group died within 24 weeks of age compared with $0 \%$ of alum-treated and control groups. Notably, vaccinated mice using alum developed comparable amounts of anti-PTX3 with those vaccinated using CFA as adjuvant. Recently, Shoenfeld Y et al. (24) described a new syndrome called ASIA and we subsequently demonstrated that CFA injection causes a syndrome resembling human ASIA in NZB/NZWF1 mice (25). In our new experiment, mice treated with CFA showed an aggravation of lupus with unrelentless progression when the disease was established, confirming the pathogenic effect of CFA for ASIA syndrome onset. By contrast, alum-injected mice demonstrated 
a delayed lupus onset to those receiving CFA, similar to the disease progression observed in PBS-injected mice. Furthermore, PTX3/alum vaccinated mice showed an immune response after vaccination resembling that of PTX3/CFA vaccinated mice. Increased risk of infection, caused by a deregulated immune response and by immunosuppressive therapy, has been observed far more in patients affected by autoimmune diseases than in healthy subjects $(1,2,7,28-31)$. Furthermore, many studies showed that not only the antigens of vaccines, but also adjuvants and excipients might enhance antigenic stimulation $(7,10,32-35)$, triggering and/or worsening autoimmune and rheumatic diseases (7-9, $32,36,37)$, also weeks or even months or years after vaccination $(13,35)$. Adjuvants may also induce the production of antibodies against themselves, as reported in US military personnel with GWS, in whom high levels of anti-squalene circulating antibodies were found $(16-18,38)$. All these negative effects are confirmed by many in vivo studies with murine and rat models $(10,19-23,35,39)$.

There is some evidence that individual susceptibility factors might play a crucial role in intolerance to adjuvants $(40,41)$. In rats, the genetic background strongly influences the size of lesions induced by intramuscular injection of alum (41). Adverse response to the adjuvants and excipients of vaccines might also depend on susceptibility genes, such as $H L A-D R B 1 * 01$, that may favor the development of autoimmune diseases (42). It has been widely demonstrated that mutations in the HLA-DR genes are related to the susceptibility and predisposition to SLE (43). Genetic predisposition, defective apoptosis and abnormalities in TLR signalling, B and T-cell tolerance, complement activation, cytokine regulation, and endothelial cell functions are all factors involved in lupus pathogenesis both in humans and in NZB/NZWF1 mice (1, $3,44)$.

CFA is the most active adjuvant because of its composition that includes components of $M$. tuberculosis. The immunization power of CFA resides mainly in the concomitant presence of 2 different antigens: the vaccine antigen and the $M$. tuberculosis components of CFA $(39,45)$. For this reason, CFA should be avoided as an adjuvant during immunization of autoimmune-prone mice in experimental studies; in addition, we suggest avoiding CFA even in non autoimmune prone mice due to its autoimmune potential (10).

By contrast, although some studies highlighted a deleterious role played by alum during vaccination $(15,16)$, our study showed that it may be safer than CFA and, in turn, it should provide a good substitute for CFA during vaccinations. Furthermore, no difference was observed in disease onset and progression between mice vaccinated with alum and mice injected with PBS, demonstrating that alum does not affect the disease.

Many other studies are required to understand the relationship among vaccines, adjuvants, and autoimmunity, in order to optimize the preparation and the use of vaccines, mainly for patients affected by autoimmune diseases.

\section{REFERENCES}

1. Doria A, Zen M, Canova M, et al. SLE diagnosis and treatment: when early is early. Autoimmun Rev. 2010; 10: 55-60.

2. Zandman-Goddard G, Peeva E, Shoenfeld Y. Gender influences SLE-immune cells, genetics, experimental models and lupus patients. Clin Exp Rheumatol. 2010; 28: 297-9.

3. Festing MFW. Inbred strains of mice. Mouse Genome. 1997; 95: 519-686.

4. Ghirardello A, Doria A, Zampieri S, et al. Antinucleosome antibodies in SLE: a two-year follow-up study of 101 patients. J Autoimmunity. 2004; 22: 235-40.

5. Jørgensen MH, Rekvig OP, Jacobsen RS, et al. Circulating levels of chromatin fragments are inversely correlated with anti-dsDNA antibody levels in huwman and murine systemic lupus erythematosus. Immunol Lett. 2011; 138: $179-86$.

6. Matrat A, Veysseyre-Balter C, Trolliet P, et al. Simultaneous detection of anti-C1q and antidouble stranded DNA autoantibodies in lupus nephritis: predictive value for renal flares. Lupus. 2011; 20: 28-34.

7. Glück T, Muller-Ladner U. Vaccination in patients with chronic rheumatic or autoimmune 
diseases. Clin Infect Dis. 2008; 46: 1459-65.

8. Pavlovic M, Kats A, Cavallo M, Shoenfeld Y. Clinical and molecular evidence for association of SLE with parvovirus B19. Lupus. 2010; 19: 783-92.

9. Pender MP. Preventing and curing multiple sclerosis by controlling Epstein-Barr virus infection. Autoimmun Rev. 2009; 8: 563-8.

10. Israeli E, Agmon-Levin N, Blank M, Shoenfeld Y. Adjuvants and autoimmunity. Lupus. 2009; 18: 217-25.

11. Eisenbarth SC, Colegio OR, O'Connor Jr W, et al. Crucial role for the nalp3 inflammasome in the immunostimulatory properties of aluminium adjuvants. Nature. 2008; 453: 1122-6.

12. Orbach H, Tanay A. Vaccines as trigger for myopathies. Lupus. 2009; 18: 1213-6.

13. Mikaeloff Y, Caridade G, Suissa S, Tardieu M. Hepatitis B vaccine and the risk of CNS inflammatory demyelination in childhood. Neurology. 2009; 72: 873-80.

14. Di Benedetto G, Pierangeli M, Scalise A, Bertani A. Paraffin oil injection in the body: an obsolete and destructive procedure. Ann Plast Sur. 2002; 49: 391-6.

15. Gherardi RK. Lessons from macrophagic myofasciitis: towards definition of a vaccine adjuvant-related syndrome. Rev Neurolog. 2003; 159: 162-4.

16. Asa PB, Cao Y, Garry RF. Antibodies to squalene in Gulf War syndrome. Exp Mol Pathol. 2000; 68: 55-64.

17. Asa PB, Wilson RB, Garry RF. Antibodies to squalene in recipients of anthrax vaccine. Exp Mol Pathol. 2002; 73: 19-27.

18. Lippi G, Targher G, Franchini M. Vaccination, squalene and anti-squalene antibodies: facts or finction? Eur J Intern Med. 2010; 21: 70-3.

19. Jiang C, Zhao ML, Scearce RM, Diaz M. Activation-induced deaminase-deficient MRL/lpr mice secrete high levels of protective antibodies against lupus nephritis. Arthritis Rheum. 2011; 63: 1086-96.

20. Jiang C, Zhao ML, Scearce RM, Diaz M. Activation-induced deaminase-deficient MRL/lpr mice secrete high levels of protective antibodies against lupus nephritis. Arthritis Rheum. 2011; 63: 1086-96.

21. Anderson PN, Potter M. Induction of plasma cell tumours in BALB-c mice with 2, 6, 10, 14-tetramethylpentadecane (pristane). Nature. 1969; 222: 994-5.

22. Satoh M, Reeves WH. Induction on lupusassociated autoantibodies in BALB/c mice by intraperitoneal injection of pristane. J Exp Med. 1994; 180: 2341-6.

23. Carlson BC, Jansson AM, Larsson A, et al. The endogenous adjuvant squalene can induce a chronic T-cell-mediated arthritis in rats. Am J Pathol. 2000; 156: 2057-65.

24. Shoenfeld Y, Agmon-Levin N. 'ASIA' - Autoimmune/inflammatory syndrome induced by adjuvants. J Autoimmun. 2011; 36: 4-8.

25. Bassi N, Luisetto R, Del Prete D, et al. Induction of the 'ASIA' syndrome in NZB/NZWF1 mice after injection of complete Freund's adjuvant (CFA). Lupus. 2012; 21: 203-9.

26. Bassi N, Ghirardello A, Blank M, et al. IgG anti-pentraxin 3 antibodies in systemic lupus erythematosus. Ann Rheum Dis. 2010; 69: 1704-10.

27. Bottazzi B, Vouret-Craviari V, Bastone A, et al. Multimer formation and ligand recognition by the long pentraxin PTX3. Similarities and differences with the short pentraxins C-reactive protein and serum amyloid $\mathrm{P}$ component. J Biol Chem. 1997; 272: 32817-23.

28. Doria A, Canova M, Tonon M, et al. Infections as triggers and complications of systemic lupus erythematosus. Autoimmun Rev. 2008; 8: 24-8.

29. Doria A, Zampieri S, Sarzi-Puttini P. Exploring the complex relationships between infections and autoimmunity. Autoimmun Rev. 2008; 8: 89-91.

30. Cooke A, Ferraccioli GF, Herrmann M, et al. Induction and protection of autoimmune rheumatic diseases: the role of infections. Clin Exp Rheumatol. 2008; 26: S1-7.

31. Girschick HJ, Guilherme L, Inman RD, et al. Bacterial triggers and rheumatic diseases. Clin Exp Rheumatol. 2008; 26: S12-7.

32. Orbach H, Agmon-Levin N, Zandman-Goddard G. Vaccines and autoimmune diseases of the adult. Discov Med. 2010; 9: 90-7.

33. Geier DA, Geier MR. A case-comtrol study of serious autoimmune adverse avents following hepatitis B immunization. Autoimmunity. 2005; 38: 295-301.

34. Abu-Shakra M. Safety of vaccination of patients with systemic lupus erythematosus. Lupus. 2009; 18: 1205-8.

35. Agmon-Levin N, Paz Z, Israeli E, Shoenfeld Y. Vaccines and autoimmunity. Nat Rev Rheumatol. 2009; 5: 648-52.

36. Wraith DC, Goldman M, Lambert PH. Vaccination and autoimmune disease: what is the evidence? Lancet. 2003; 362: 1659-66.

37. Schattner A. Consequence or coincidence? The occurrence, pathogenesis and significance of autoimmune manifestations after viral vaccines. Vaccine. 2005; 23: 3876-86.

38. Hornung V, Bauernfeind F, Halle A, et al. Silica crystals and aluminum salts activate the NALP3 inflammasome through phagosomal destabilization. Nat Immunol. 2008; 9: 84756.

39. Kluger N, Cohen P, Fallet-Bianco C, Guillevin L. Mycobacterium chelonae infection under adalimumab therapy for spondylarthritis. Clin Exp Rheumatol. 2010; 28: 101-2.

40. Gherardi RK, Authier FJ. Macrophagic myofasciitis: characterization and pathophysiology. Lupus. 2012; 21: 184-9. 
41. Authier FJ, Sauvat S, Christov C, et al. AlOH3-adjuvanted vaccine-induced macrophagic myofasciitis in rats is influenced by the genetic background. Neuromuscul Disord. 2006; 16: 347-52.

42. Guis S, Pellissier JF, Nicoli F, et al. HLADRB $1 * 01$ and macrophagic myofasciitis. Arthritis Rheum. 2002; 46: 2535-7.

43. Fu SM, Deshmukh US, Gaskin F. Pathogenesis of systemic lupus erythematosus revisited 2011: end organ resistance to damage, autoan- tibody initiation and diversification, and HLADR. J Autoimmun. 2011; 37: 104-12.

44. Gomez-Martin D, Diaz-Zamudio M, Crispin JC, Alcocer-Varela J. Interleukin 2 and systemic lupus erythematosus: beyond the transcriptional regulatory net abnormalities. Autoimmun Rev. 2009; 9: 34-9.

45. Yazisiz V, Erbasan F, Inan D, et al. Bone marrow infection caused by Mycobacterium avium complex in a patient with systemic lupus erythematosus. Lupus. 2010; 19: 323-6. 